\title{
Reciprocity based transmission line equations for higher order eigenmodes in lossy waveguides
}

\author{
F. Olyslager*, A. Franchois, D. De Zutter \\ Department of Information Technology, Ghent University, Sint-Pietersnieuwstraat 41, B-9000 Ghent, Belgium
}

Received 25 November 2003; received in revised form 22 April 2004; accepted 26 April 2004

Available online 29 September 2004

\begin{abstract}
An important branch in electromagnetic research is the construction of circuit models for devices starting from electromagnetic field simulations. Among the first of such descriptions was the transmission line representation of TEM fields along a coaxial cable, the so-called telegrapher's equations. Although the transmission line representation directly follows from Maxwell's equations only in the pure TEM and, at low frequencies, also in the quasi-TEM situations, many authors have published representations that are valid for the propagation at arbitratry frequencies in general waveguides. These representations usually are based on the assumption that equal complex power is propagated in both the waveguide and the transmission line. However, this leads to problems regarding the reciprocity of such transmission line representations. About 10 years ago Prof. de Hoop suggested the present authors a solution to these problems. In honor of the celebration of Prof. de Hoop's 75th anniversary, we present in this paper these "reciprocity based transmission line representations" in a new and concise manner and apply them to a new problem dealing with higher order eigenmodes in single conductor waveguides. It is shown that again reciprocity leads to simple and hence esthetically pleasing results.
\end{abstract}

(C) 2004 Elsevier B.V. All rights reserved.

PACS: 41.20.Jb; 42.79.Gn

Keywords: Transmission lines; Waveguides; Coaxial cable; Reciprocity

\section{Introduction}

In the mid of the 19th century the first submarine telegrapher cables were installed, first between Calais and Dover and later across the Atlantic. These cables were coaxial cables with the sea water acting as the return conductor. Actually, the first succesful submarine cable of 1851 from Dover to Calais was a multiconductor coaxial cable with

\footnotetext{
* Corresponding author. Tel.: +32 92643344 ; fax: +32 92643593.

E-mail address: olyslag@intec.ugent.be (F. Olyslager).
} 
four inner conductors. Due to the use of ever longer cables the need arose for a mathematical description of the propagation of currents and voltages. Lord Kelvin applied Ohm's and Kirchhoff's laws to an infinitesimal length of the line to obtain the equations

$$
\begin{aligned}
& \frac{\partial}{\partial z} v(z, t)=-R i(z, t), \\
& \frac{\partial}{\partial z} i(z, t)=-C \frac{\partial}{\partial t} v(z, t) .
\end{aligned}
$$

Eliminating the current yields a parabolic type of equation. In 1874 Oliver Heaviside corrected Eqs. (1) and (2) by first introducing the conductance $G$ of the insulation and finally in 1876 by adding the inductance $L$ to obtain the famous telegrapher's equations [1]

$$
\begin{aligned}
& \frac{\partial^{2}}{\partial z^{2}} i(z, t)-L C \frac{\partial^{2}}{\partial t^{2}} i(z, t)-(R C+G L) \frac{\partial}{\partial t} i(z, t)-G R i(z, t)=0, \\
& \frac{\partial^{2}}{\partial z^{2}} v(z, t)-L C \frac{\partial^{2}}{\partial t^{2}} v(z, t)-(R C+G L) \frac{\partial}{\partial t} v(z, t)-G R v(z, t)=0 .
\end{aligned}
$$

A few years later these equations were verified using Maxwell's equations.

Both electromagnetic waveguides and transmission lines are structures that guide waves in one direction. Oliver Heaviside proved that the propagation of TEM electromagnetic waves along a coaxial cable can be described by the propagation of current and voltage waves along a transmission line. This is easily generalised to the TEM fields in the so-called homogeneous multiconductor waveguides, i.e. waveguides consisting of a number of parallel perfect conductors embedded in a homogeneous isotropic dielectric. In this case the coupled transmission line equations follow unambiguously from Maxwell's equations.

In the 1970s and early 1980s this was generalised to inhomogeneous multiconductor waveguides. In this case the analysis was limited to low frequency where the fields are still quasi-TEM. By using a low frequency expansion, Maxwell's equations still reduce to the transmission line equations [2,3]. The quasi-TEM fields correspond to the lowest order or fundamental eigenmodes in the waveguide. These eigenmodes have no cut-off frequencies and at low frequencies the longitudinal field components along the propagation direction become negligible compared to the transverse components.

With the use of microwave circuits at higher frequencies, the quasi-TEM assumption became questionable. In the full-wave regime the non-negligible longitudinal field components impede an unambiguous deriviation of the transmission line equations from Maxwell's equations. The reason being that current and voltage in the waveguide loose their strict meaning. Nevertheless, the analogy between a waveguide and a transmission line and the need for circuit descriptions resulted in the construction of several transmission line representations valid in the full-wave regime. These models are based on the assumption that the same complex power is propagated in the waveguide and the transmission line. The first attempts [4,5] faced some inconsistencies that were remedied in [6]. Different power based transmission line representations were built, so-called PI-models, where the current on the transmission line has a physical meaning, and PV-models, where the voltage has a physical meaning.

Application of these new transmission line representations to multiconductor waveguides involving losses resulted in a set of coupled transmission lines that was no longer reciprocal, whereas the original waveguide is composed of reciprocal media only. This was noticed by the first author and taken as a fact. However, Prof. de Hoop, having carefully read the $\mathrm{PhD}$ [7] of the first author, suggested a new transmission line representation, which is not based on the conservation of complex power but on the orthogonality of the eigenmodes. We call these reciprocity based transmission line models. Also in this case one can distinguish between RI-models and RV-models. These new results were published in [8]. For lossless waveguides or at low frequencies there is no difference between the power and reciprocity based transmission line models. 
The publication [8] was the onset of extensive research to generalise the theory to non-isotropic waveguides [9]. First anisotropic waveguides were considered and later bianisotropic waveguides leading to the concept of bitransmission lines. Also the inclusion in the transmission line model of external fields incident on the waveguide [10] and the representation of waveguide discontinuities [8] were investigated. All these results are summarized in a monography [11] which was a direct consequence of Prof. de Hoop's comments on [7].

As implicitly mentioned above, transmission line models are usually applied for to describe the fundamental eigenmodes in multiconductor waveguides. In this contribution we wish to apply the power and reciprocity based transmission line models to higher order eigenmodes in a single conductor waveguide, i.e. a waveguide with one signal conductor and a reference conductor, such as the coaxial cable. We will show that the above mentioned transmission line models remain valid but that extra degrees of freedom are available. In particular it will be shown, exploiting these extra degrees of freedom, that reciprocity based models can be constructed in such a way that the set of coupled transmission lines become decoupled.

As an example we will apply the theory to a coaxial cable with a lossy core referring to the original submarine telegrapher cables that led to the telegrapher's equations.

In the first part of the paper we will present the power and reciprocity based transmission line models. This theory obviously is not new but we will present it in a slightly different and more efficient manner than e.g. in [8]. Next we adapt the theory to higher order eigenmodes in a single conductor waveguide and we finally illustrate the technique with the analysis of the lossy coaxial cable.

\section{Theory}

We assume that all fields, voltages and currents have the same time-harmonic $\mathrm{e}^{\mathrm{j} \omega t}$ dependence and we orient the $z$-axis along the propagation or longitudinal direction of the waveguides and transmission lines.

We restrict the analysis to waveguides filled with inhomogeneous isotropic media characterised by a permittivity $\epsilon(\boldsymbol{\rho})$ and permeability $\mu(\boldsymbol{\rho})$, where $\boldsymbol{\rho}=x \mathbf{u}_{x}+y \mathbf{u}_{y}$. The fields in a waveguide can be expanded in the set of eigenmodes of the waveguide [11]. Let us write the fields of an eigenmode as

$$
\mathbf{e}(\mathbf{r})=\mathbf{E}(\boldsymbol{\rho}) \mathrm{e}^{-\gamma^{\mathrm{w}} \mathrm{z}}, \quad \mathbf{h}(\mathbf{r})=\mathbf{H}(\boldsymbol{\rho}) \mathrm{e}^{-\gamma^{\mathrm{w}} \mathrm{z}},
$$

where $\gamma^{w}$ is the propagation coefficient of the eigenmode. The considered waveguides are bidirectional [12] meaning that the eigenmodes come into pairs, one with propagation coefficient $\gamma^{w}$ and another with propagation coefficient $-\gamma^{w}$. For two eigenmodes with indices $i$ and $j$ we define the integral

$$
R_{i j}^{w}=\frac{1}{2} \int_{S}\left[\mathbf{E}_{i}(\boldsymbol{\rho}) \times \mathbf{H}_{j}(\boldsymbol{\rho})\right] \cdot \mathbf{u}_{z} \mathrm{~d} S,
$$

where $S$ is the cross-section of the waveguide. The orthogonality of the eigenmodes in a waveguide yields that $R_{i j}^{w}=0$ when $i \neq j$, or more correctly when $\gamma_{i}^{w} \neq \gamma_{j}^{w}$. From now on we will neglect degenerate eigenmodes with $\gamma_{i}^{w}=\gamma_{j}^{w}$ for $i \neq j$. However, using e.g. a Gram-Schmidt procedure, the theory can be extended to include degeneracy of eigenmodes as well. The complex cross-power $P_{i j}^{w}$ between two eigenmodes is defined as

$$
P_{i j}^{w}=\frac{1}{2} \int_{S}\left[\mathbf{E}_{i}(\boldsymbol{\rho}) \times \mathbf{H}_{j}^{*}(\boldsymbol{\rho})\right] \cdot \mathbf{u}_{z} \mathrm{~d} S .
$$

In general $P_{i j}^{w} \neq 0$ when $i \neq j$. However, in a lossless waveguide, see [11], one can choose the transverse parts of $\mathbf{E}_{i}(\boldsymbol{\rho})$ and $\mathbf{H}_{j}(\boldsymbol{\rho})$ to be real such that $P_{i j}^{w}=R_{i j}^{w}$, meaning power orthogonality. A set of coupled transmission lines is described in time-harmonic regime by

$$
\frac{\mathrm{d}}{\mathrm{d} z} \mathbf{v}(z)=-\overline{\bar{Z}} \cdot \mathbf{i}(z),
$$




$$
\frac{\mathrm{d}}{\mathrm{d} z} \mathbf{i}(z)=-\overline{\bar{Y}} \cdot \mathbf{v}(z)
$$

with $\mathbf{v}(z)$ and $\mathbf{i}(z)$ vectors grouping the voltages and currents of the different lines and with $\overline{\bar{Y}}$ and $\overline{\bar{Z}}$ the circuit admittance and impedance matrices, respectively, also separated in a real and imaginary part as $\overline{\bar{Y}}=\mathrm{j} \omega \overline{\bar{C}}+\overline{\bar{G}}$ and $\overline{\bar{Z}}=\mathrm{j} \omega \overline{\bar{L}}+\overline{\bar{R}}$, with $\overline{\bar{C}}$ the capacitance matrix, $\overline{\bar{G}}$ the conductance matrix, $\overline{\bar{L}}$ the inductance matrix and $\overline{\bar{R}}$ the resistance matrix. The eigenmodes in the coupled transmission lines are

$$
\mathbf{v}(z)=\mathbf{V} \mathrm{e}^{-\gamma^{\mathrm{l}} \mathrm{z}}, \quad \mathbf{i}(\mathrm{z})=\mathbf{I} \mathrm{e}^{-\gamma^{\mathrm{l}} \mathrm{z}},
$$

where $\mathbf{V}$ and $\mathbf{I}$ are the modal voltages and currents. Substitution of (10) in (8) and (9) yields that

$$
\begin{aligned}
& -\gamma^{l} \mathbf{V}=-\overline{\bar{Z}} \cdot \mathbf{I}, \\
& -\gamma^{l} \mathbf{I}=-\overline{\bar{Y}} \cdot \mathbf{V} .
\end{aligned}
$$

It is immediately obvious that also here for an eigenmode with propagation coefficient $\gamma^{l}$ there exists an eigenmode with propagation coefficient $-\gamma^{l}$. For two eigenmodes with indices $i$ and $j$ we define

$$
R_{i j}^{l}=\frac{1}{2} \mathbf{V}_{i}^{\mathrm{T}} \cdot \mathbf{I}_{j}
$$

When $i \neq j, R_{i j}^{l}=0$ due to the orthogonality of the eigenmodes [11]. The cross-power $P_{i j}^{l}$ between two eigenmodes is given by

$$
P_{i j}^{l}=\frac{1}{2} \mathbf{V}_{i}^{\mathrm{T}} \cdot \mathbf{I}_{j}^{*}
$$

In general $P_{i j}^{l} \neq 0$ when $i \neq j$. For a lossless coupled set of transmission lines, i.e. when $\overline{\bar{Z}}^{\mathrm{T}}=-\overline{\bar{Z}}^{*}$ and $\overline{\bar{Y}}^{\mathrm{T}}=-\overline{\bar{Y}}^{*}$, $\mathbf{V}_{i}$ and $\mathbf{I}_{i}$ can be chosen real such that $P_{i j}^{l}=R_{i j}^{l}$.

We will now construct a coupled transmission line model for a set of eigenmodes in a waveguide. This means that we will derive expressions for the transmission line parameters $\overline{\bar{Y}}$ and $\overline{\bar{Z}}$ as a function of the modal fields in the waveguide. $N$ eigenmodes in the waveguide are represented by a set of $N$ coupled transmission lines.

First we demand that arbitrary waves composed from the considered eigenmodes in both the waveguide and the transmission line have the same velocities. Therefore, it is needed that $\gamma_{i}^{l}=\gamma_{i}^{w}$, with $i=1,2, \ldots, N$. If we group the propagation coefficients on the diagonals of two diagonal matrices $\overline{\bar{\gamma}}^{l}$ and $\overline{\bar{\gamma}}^{w}$, then this means that

$$
\overline{\bar{\gamma}}^{l}=\overline{\bar{\gamma}}^{w} \text {. }
$$

A second requirement is that the same complex power be propagated in the waveguide and the set of coupled transmission lines. This is guaranteed by expressing that

$$
\overline{\bar{P}}^{l}=\overline{\bar{P}}^{w},
$$

where $\overline{\bar{P}}^{l}$ and $\overline{\bar{P}}^{w}$ are $N \times N$ matrices constructed from $P_{i j}^{l}$ and $P_{i j}^{w}$.

When grouping the modal currents $\mathbf{I}_{i}$ and $\mathbf{V}_{i}, i=1,2, \ldots, N$, columnwise in matrices $\overline{\bar{I}}$ and $\overline{\bar{V}}$, then it follows from (11) and (12) that

$$
\begin{aligned}
& \overline{\bar{Z}}=\overline{\bar{V}} \cdot \overline{\bar{\gamma}}^{l} \cdot \overline{\bar{I}}^{-1}, \\
& \overline{\bar{Y}}=\overline{\bar{I}} \cdot \overline{\bar{\gamma}}^{l} \cdot \overline{\bar{V}}^{-1} .
\end{aligned}
$$




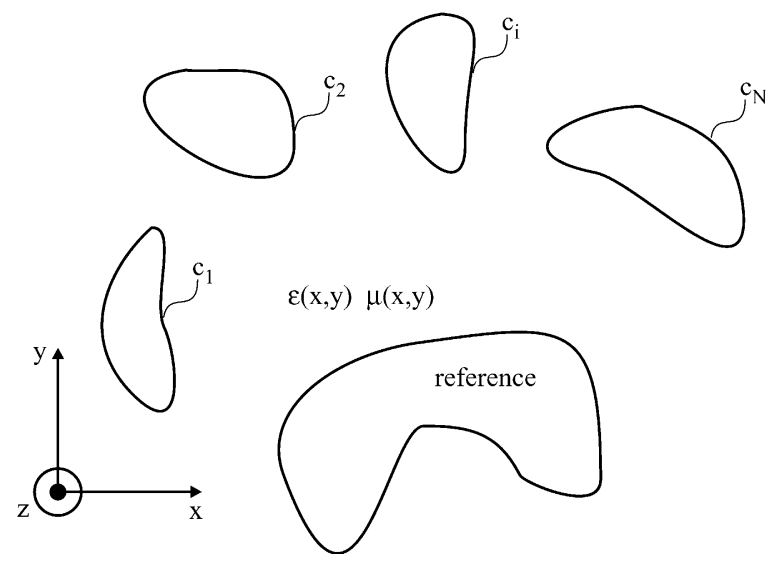

Fig. 1. Cross-section of a multiconductor waveguide with $N$ conductors and a reference conductor.

Using (15) and $\overline{\bar{P}}^{l}=(1 / 2) \overline{\bar{V}}^{\mathrm{T}} \cdot \overline{\bar{I}}^{*}=\overline{\bar{P}}^{w}$ this can be written as

$$
\begin{aligned}
& \overline{\bar{Z}}=2\left(\overline{\bar{I}}^{\mathrm{T} *}\right)^{-1} \cdot\left(\overline{\bar{P}}^{w}\right)^{\mathrm{T}} \cdot \overline{\bar{\gamma}}^{w} \cdot \overline{\bar{I}}^{-1}, \\
& \overline{\bar{Y}}=\frac{1}{2} \overline{\bar{I}} \cdot \overline{\bar{\gamma}}^{w} \cdot\left[\left(\overline{\bar{P}}^{w}\right)^{\mathrm{T}}\right]^{-1} \cdot \overline{\bar{I}}^{\mathrm{T} *} .
\end{aligned}
$$

The parameters $\overline{\bar{Y}}$ and $\overline{\bar{Z}}$ are fully defined when the matrices $\overline{\bar{I}}$ have been determined.

Let us now make the assumption that we have a multiconductor waveguide with $N$ signal conductors and one reference conductor (Fig. 1) and that we consider the $N$ fundamental eigenmodes that have no cut-off frequency, i.e. that exist for all frequencies. An obvious choice for the elements of $\overline{\bar{I}}$ is to assign to them the currents flowing in each conductor for each eigenmode, i.e.

$$
I_{i j}=\oint_{c_{j}} \mathbf{H}_{i}(\boldsymbol{\rho}) \cdot \mathrm{d} \mathbf{l},
$$

where $i, j=1,2, \ldots, N$ and $c_{j}$ is the boundary of the $j$ th conductor. In this way all quantities on the right-hand side of (19) and (20) follow from quantities in the waveguide. The transmission line model constructed in this way is called the PI-model. It is also possible to relate $\overline{\bar{V}}$ to voltages in the waveguide. In this way a PV-model is constructed. We will not pursue voltage based models but rather refer to [8] and [11] for more information on these.

A reciprocal transmission line is characterised by symmetric $\overline{\bar{Y}}$ and $\overline{\bar{Z}}$ matrices. However, the expressions (19) and (20) do not indicate that $\overline{\bar{Y}}$ and $\overline{\bar{Z}}$ indeed are symmetric. On the contrary numerical examples [8,13] have shown that in general $\overline{\bar{Y}}$ and $\overline{\bar{Z}}$ are not symmetric. Prof. de Hoop suggested a way out of this problem by dropping the demand that the same complex power be propagated in the waveguide and the set of coupled transmission lines. Instead, it is required that $\overline{\bar{R}}^{l}=\overline{\bar{R}}^{w}$. This then yields the following expressions for the transmission line parameters

$$
\begin{aligned}
& \overline{\bar{Z}}=2\left(\overline{\bar{I}}^{\mathrm{T}}\right)^{-1} \cdot\left(\overline{\bar{R}}^{w}\right)^{\mathrm{T}} \cdot \overline{\bar{\gamma}}^{w} \cdot \overline{\bar{I}}^{-1}, \\
& \overline{\bar{Y}}=\frac{1}{2} \overline{\bar{I}} \cdot \overline{\bar{\gamma}}^{w} \cdot\left[\left(\overline{\bar{R}}^{w}\right)^{\mathrm{T}}\right]^{-1} \cdot \overline{\bar{I}}^{\mathrm{T}} .
\end{aligned}
$$

Since, $\overline{\bar{R}}^{w}$ is a diagonal matrix it follows that $\overline{\bar{Y}}$ and $\overline{\bar{Z}}$ are symmetric. 


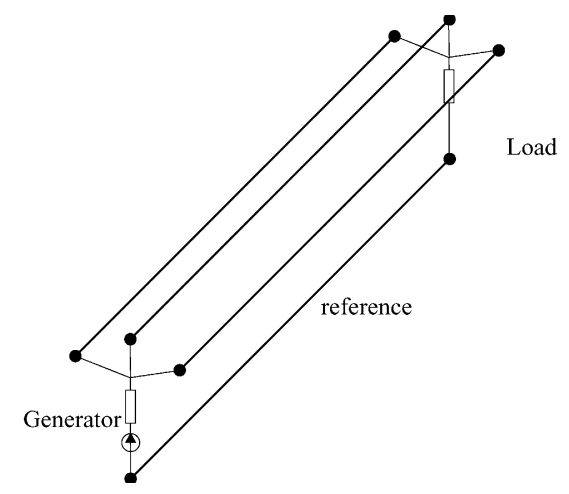

Fig. 2. A set of coupled transmission lines with all lines connected at the load and generator.

Let us now consider a single conductor waveguide, i.e. a waveguide with one signal conductor and a reference conductor. Such a waveguide will have one eigenmode that exists for all frequencies. We wish to construct a transmission line model that incorporates not only this eigenmode but also higher order eigenmodes. For $N$ eigenmodes a set of $N$ coupled transmission lines will be derived. Eqs. (19) and (20) or (22) and (23) are still applicable to this case. However, it is not clear how to define the elements of $\overline{\bar{I}}$ since there is only one signal conductor and not $N$ as in (21). We can still demand that the total current on the signal conductor in the waveguide is equal to the sum of all the currents on all the lines in the transmission line, i.e.

$$
\sum_{j=1}^{N} I_{i j}=\oint_{c} \mathbf{H}_{i}(\boldsymbol{\rho}) \cdot \mathrm{d} \mathbf{l},
$$

where $c$ is the boundary of the signal conductor. This assumes that at the load and generator of the set of coupled transmission lines all the signal conductors are connected with each other as is shown in Fig. 2. Obviously (24) defines only $N$ conditions on $\overline{\bar{I}}$, leaving many degrees of freedom. A particular simple choice is to make $\overline{\bar{I}}$ a diagonal matrix such that

$$
I_{i i}=\oint_{c} \mathbf{H}_{i}(\rho) \cdot \mathrm{d} \mathbf{l}
$$

From (22) and (23) it then follows that $\overline{\bar{Y}}$ and $\overline{\bar{Z}}$ are diagonal matrices, i.e. that the set of transmission lines becomes a decoupled set of equations. Note that $\overline{\bar{Y}}$ and $\overline{\bar{Z}}$ following from (19) and (20) will not be diagonal.

The simple choice (25) is only permisable as long as the integral (25) does not vanish. This would lead to a singular $\overline{\bar{Z}}$-matrix as it need the inverse of $\overline{\bar{I}}$. In (21) the problem of the existence of $\overline{\bar{I}}^{-1}$ poses less of a problem. Indeed, at low frequencies a quasi-TEM analysis directly leeds to the existence of $\overline{\bar{Y}}$ and $\overline{\bar{Z}}$ indicating the existence of $\overline{\bar{I}}^{-1}$. Of course, it cannot be guaranteed that higher frequencies exist for which $\overline{\bar{I}}^{-1}$ is singular.

\section{Coaxial cable}

As an example we consider a coaxial cable with a lossy conductor core. The coaxial structure allows an analytical treatment. We will consider the geometry of Fig. 3 consisting of a core cylinder with radius $a$ and complex medium parameters $\epsilon_{2}$ and $\mu_{2}$ surrounded by another cylindrical medium with complex parameters $\epsilon_{1}$ and $\mu_{1}$ and radius $b$. The whole structure is enclosed by a perfect electric conducting shield. In the limit for $\epsilon_{2} \rightarrow-\mathrm{j} \infty$ and $\epsilon_{1}$ and $\mu_{1}$ 


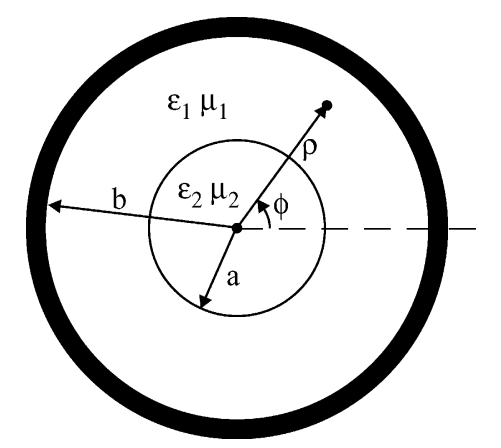

Fig. 3. Geometry of a coaxial cable with a lossy core.

real a lossless coaxial cable with a perfect electric conducting core is obtained. We will limit the discussion to fields that only depend on the radial coordinate. In this case the eigenmodes of the structure still fall apart in TE and TM eigenmodes. We will only consider the analysis of the TM eigenmodes.

Using polar coordinates as shown in Fig. 3, the TM modal field components $E_{z}(\rho), E_{\rho}(\rho)$ and $H_{\phi}(\rho)$ satisfy

$$
\frac{1}{\rho} \frac{\partial}{\partial \rho}\left[\rho \frac{\partial}{\partial \rho} E_{z}(\rho)\right]+\kappa_{i}^{2} E_{z}(\rho)=0
$$

with $\kappa_{i}^{2}=\omega^{2} \epsilon_{i} \mu_{i}+\left(\gamma^{w}\right)^{2}$ and

$$
E_{\rho}(\rho)=-\frac{\gamma^{w}}{\kappa_{i}^{2}} \frac{\partial}{\partial \rho} E_{z}(\rho), \quad H_{\phi}(\rho)=-\frac{\mathrm{j} \omega \epsilon_{i}}{\kappa_{i}^{2}} \frac{\partial}{\partial \rho} E_{z}(\rho),
$$

with $i=1,2$. By writing the general solution of (26) in both layers and imposing the appropriate boundary conditions one finds the following transcendental dispersion relation for the propagation coefficients

$$
J_{0}\left(\kappa_{1} a\right) Y_{0}\left(\kappa_{1} b\right)-J_{0}\left(\kappa_{1} b\right) Y_{0}\left(\kappa_{1} a\right)-\frac{\kappa_{2} \epsilon_{1} J_{0}\left(\kappa_{2} a\right)}{\kappa_{1} \epsilon_{2} J_{1}\left(\kappa_{2} a\right)}\left[J_{1}\left(\kappa_{1} a\right) Y_{0}\left(\kappa_{1} b\right)-J_{0}\left(\kappa_{1} b\right) Y_{1}\left(\kappa_{1} a\right)\right]=0 .
$$

The modal field component $E_{z, j}(\rho)$ for an eigenmode with propagation coefficient $\gamma_{j}$ can be written as

$$
E_{z, j}(\rho)=\frac{J_{0}\left(\kappa_{1, j} \rho\right)-J_{0}\left(\kappa_{1, j} b\right)}{Y_{0}\left(\kappa_{1, j} b\right)} Y_{0}\left(\kappa_{1, j} \rho\right),
$$

for $a<\rho<b$ and as

$$
E_{z, j}(\rho)=\frac{J_{0}\left(\kappa_{1, j} a\right) Y_{0}\left(\kappa_{1, j} b\right)-J_{0}\left(\kappa_{1, j} b\right) Y_{0}\left(\kappa_{1, j} a\right)}{Y_{0}\left(\kappa_{1, j} b\right) J_{0}\left(\kappa_{2, j} a\right)} J_{0}\left(\kappa_{2, j} \rho\right)
$$

for $\rho<a$. The other field components $E_{\rho, j}(\rho)$ and $H_{\phi, j}(\rho)$ are obtained from $E_{z, j}(\rho)$ through (27).

As signal conductor we use the outer conductor. The total current on this outer conductor for eigenmode $i$ is given by

$$
I_{i i}=-\frac{4 \mathrm{j} \omega \epsilon_{1}}{\left(\kappa_{1, i}\right)^{2} Y_{0}\left(\kappa_{1, i} b\right)} .
$$

The modal cross-power between eigenmodes $i$ and $j$ is given by

$$
P_{i j}^{w}=\pi \int_{0}^{b} E_{\rho, i}(\rho) H_{\phi, j}^{*}(\rho) \rho \mathrm{d} \rho .
$$




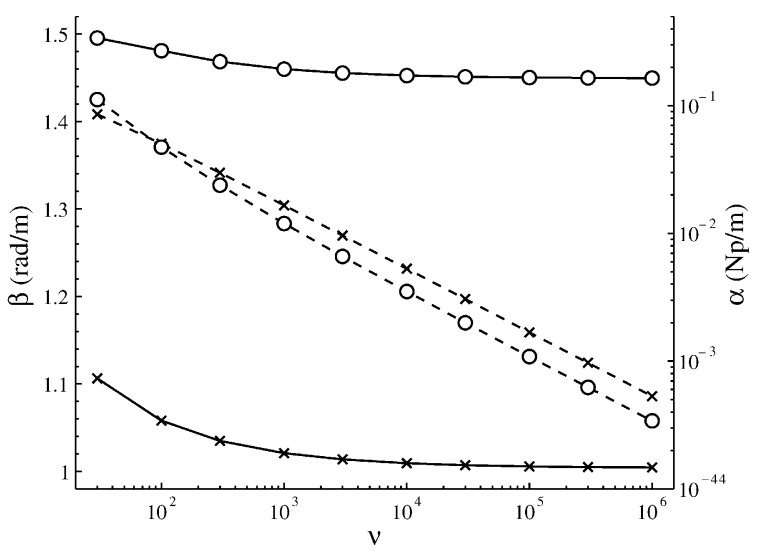

Fig. 4. Real ( $\alpha$ ) (dashed lines) and imaginary $(\beta)$ (solid lines) part of the normalised propagation coefficients $\gamma / k_{0}$ for two eigenmodes in the coaxial cable of Fig. 3 as a function of the parameter $v\left(\epsilon_{2}=-\mathrm{j} v \epsilon_{0}\right)((\bigcirc)$ fundamental eigenmode, $(\times)$ higher order eigenmode $)$.

The diagonal elements of $\overline{\bar{R}}^{w}$ are given by

$$
R_{i i}^{w}=\pi \int_{0}^{b} E_{\rho, i}(\rho) H_{\phi, i}(\rho) \rho \mathrm{d} \rho .
$$

Both the integrals (32) and (33) require numerical evaluation.

Let us now investigate some numerical results for a cable with $a=0.0059 \mathrm{~m}, b=0.02 \mathrm{~m}, \epsilon_{1}=2.1 \epsilon_{0}$ and $\mu_{1}=\mu_{2}=\mu_{0}$, where $\epsilon_{0}$ and $\mu_{0}$ are the vacuum permittivity and permeability, respectively. The permittivity of the core is given by $\epsilon_{2}=-\mathrm{j} v \epsilon_{0}$, with $v$ a parameter that we will vary. For $v=+\infty$ the structure becomes a coaxial cable with a perfect conducting core. We chose a frequency of $10 \mathrm{GHz}$ and we consider the fundamental eigenmode and one higher order eigenmode. When the core becomes perfectly conducting the fundamental eigenmode reduces to the classical TEM eigenmode of a coaxial cable.

In Fig. 4 the normalised propagation coefficients $\gamma_{i} / k_{0}=\alpha_{i}+\mathrm{j} \beta_{i}$, with $k_{0}=\omega \sqrt{\epsilon_{0} \mu_{0}}$, of the two considered eigenmodes are plotted as a function of the parameter $v$. When $v$ increases the real parts of $\gamma_{i}$ decrease due to a

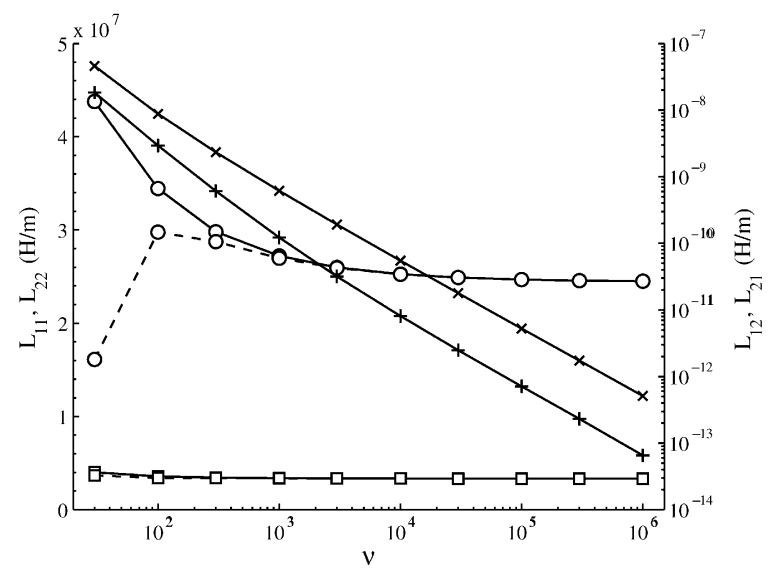

Fig. 5. Inductance matrix obtained from the PI-model (solid lines) and RI-model (dashed lines) for two eigenmodes in the coaxial cable of Fig. 3 as a function of the parameter $v\left(\epsilon_{2}=-\mathrm{j} v \epsilon_{0}\right)\left((\bigcirc) L_{11},(\square) L_{22},(\times) L_{21},(+) L_{12}\right)$. 


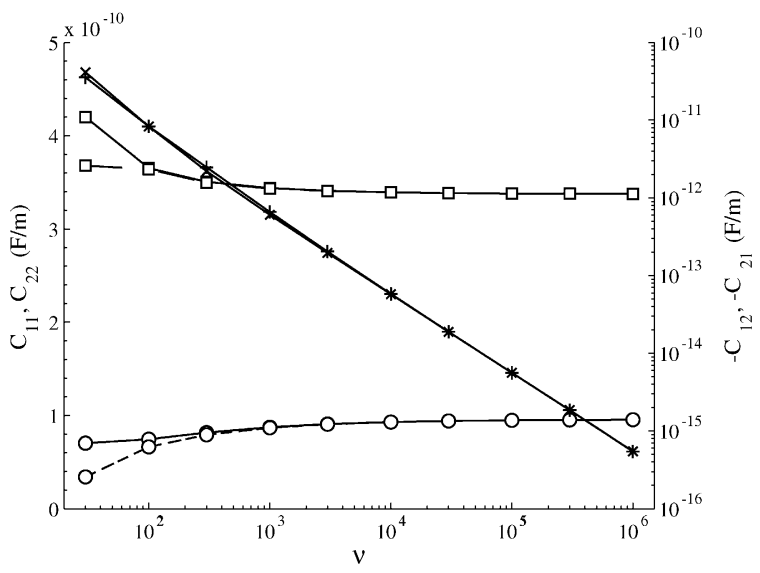

Fig. 6. Capacitance matrix obtained from the PI-model (solid lines) and RI-model (dashed lines) for two eigenmodes in the coaxial cable of Fig. 3 as a function of the parameter $v\left(\epsilon_{2}=-\mathrm{j} v \epsilon_{0}\right)\left((\bigcirc) C_{11},(\square) C_{22},(\times) C_{21},(+) C_{12}\right)$.

decrease of the losses. Figs. 5-7 show the elements of the $\overline{\bar{L}}, \overline{\bar{C}}$ and $\overline{\bar{R}}$ matrices in the PI-model as a function of the parameter $v$. The non-reciprocity of this transmission line model is clearly visible. When $v$ becomes large the elements $L_{11}$ and $C_{11}$ reach their respective values of a coaxial cable given by

$$
L=\frac{\mu \log (b / a)}{2 \pi}=244.2 \mathrm{nH} / \mathrm{m}, \quad C=\frac{2 \pi \epsilon}{\log (b / a)}=95.65 \mathrm{pF} / \mathrm{m}
$$

and the elements of $\overline{\bar{R}}$ decrease. Note that the off-diagonal elements of $\overline{\bar{L}}$ and $\overline{\bar{C}}$ also decrease. In Figs. 5-7 also the elements of the diagonal $\overline{\bar{L}}, \overline{\bar{C}}$ and $\overline{\bar{R}}$ matrices in the RI-model as a function of the parameter $v$ are shown. For large values of $v$ the difference between the RI-model and the PI-model disappears.

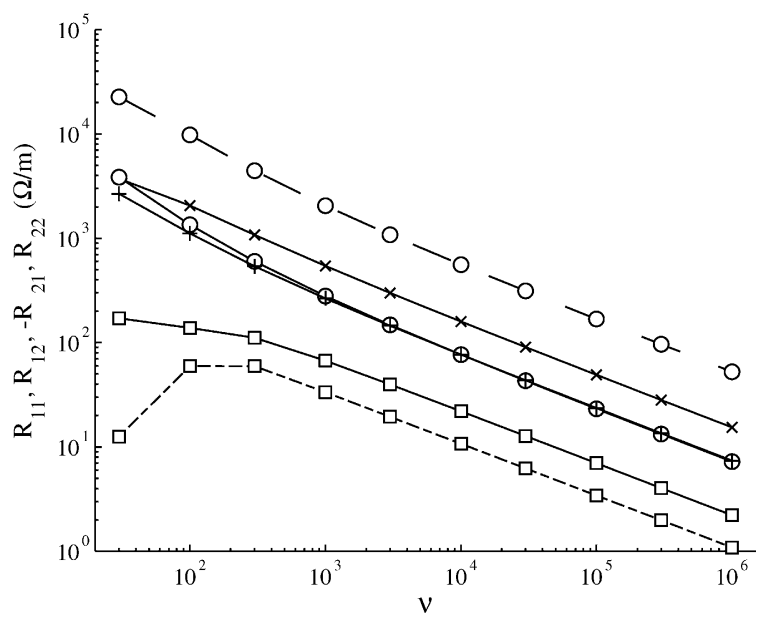

Fig. 7. Resistance matrix obtained from the PI-model (solid lines) and RI-model (dashed lines) for two eigenmodes in the coaxial cable of Fig. 3 as a function of the parameter $v\left(\epsilon_{2}=-\mathrm{j} v \epsilon_{0}\right)\left((\bigcirc) R_{11},(\square) R_{22},(\times) R_{21},(+) R_{12}\right)$. 


\section{Conclusions}

In this paper we have extended transmission line models for the fundamental eigenmodes in multiconductor waveguides to higher order eigenmodes in single conductor waveguides. We have considered power based and reciprocity based representations and have shown that reciprocity - as was advocated by Prof. de Hoop - results in the most natural, consistent and simple transmission line equations.

\section{References}

[1] P.J. Nahin, Oliver Heaviside: Sage in Solitude, The Life, Work and Times of An Electrical Genius of the Victorian Age, IEEE Press, New York, 1988.

[2] A.F. Dos Santos, J.P. Figanier, The method of series expansion in the frequency domain applied to multidielectric transmission lines, IEEE Trans. Microwave Theory Tech. 23 (1975) 753-756.

[3] I.V. Lindell, On the quasi-TEM modes in inhomogeneous multiconductor transmission lines, IEEE Trans. Microwave Theory Tech. 29 (1981) 812-817.

[4] J.R. Brews, Transmission line models for lossy waveguide interconnections in VLSI, IEEE Trans. Electron. Devices 33 (1986) $1356-1365$.

[5] R.H. Jansen, Unified user-oriented computation of shielded, covered and open planar microwave and millimeter-wave transmission-line characteristics, Microwave Opt. Acoust. 3 (1979) 14-22.

[6] N. Faché, D. De Zutter, New high-frequency circuit model for coupled lossless and lossy waveguide structures, IEEE Trans. Microwave Theory Tech. 38 (1990) 252-259.

[7] F. Olyslager, Elektromagnetische Modellering van Elektrische en Diëlektrische Golfgeleiders in Gelaagde Media (Electromagnetic modelling of electric and dielectric waveguides in layered media), PhD Thesis, Ghent, 1993.

[8] F. Olyslager, D. De Zutter, A.T. de Hoop, New reciprocal circuit model for lossy waveguide structures based on the orthogonality of the eigenmodes, IEEE Trans. Microwave Theory Tech. 42 (12) (1994) 2261-2269.

[9] F. Olyslager, Properties of and generalized full-wave transmission line models for (bi)(an)isotropoc waveguides, IEEE Trans. Microwave Theory Tech. 44 (1996) 2064-2075.

[10] D. De Zutter, F. Olyslager, High-frequency reciprocity-based circuit model for the incidence of electromagnetic waves on general waveguide structures, IEEE Trans. Microwave Theory Tech. 43 (1995) 1826-1833.

[11] F. Olyslager, Electromagnetic Waveguides and Transmission Lines, Oxford University Press, Oxford, 1999.

[12] P.R. McIsaac, Mode orthogonality in reciprocal and nonreciprocal waveguides, IEEE Trans. Microwave Theory Tech. 39 (1991) $1808-1816$.

[13] D.F. Williams, F. Olyslager, Modal cross power in quasi-TEM transmission lines, IEEE Microwave Guided Wave Lett. 6 (1996) $413-415$. 